\title{
Assessment of Physical Activity among Young Students of Different Institutes; A Multicenter Experience from A Developing Country
}

\author{
Syeda Beenish Bareeqa ${ }^{1 *}$, Syed Ijlal Ahmed ${ }^{2}$, Syeda Sana Samar ${ }^{3}$, Allahyar ${ }^{4}$ and Muhammad Tayyab Shera \\ ${ }^{1}$ Medical Student, Jinnah Medical and Dental College, Pakistan \\ ${ }^{2}$ Medical Graduate, Liaquat National Medical College and Hospital, Karachi \\ ${ }^{3}$ Medical Student, Jinnah Sindh Medical University, Pakistan \\ ${ }^{4}$ Medical House Officer, Sandeman Provincial Hospital, Pakistan \\ ${ }^{5}$ Medical House Officer, Mayo Hospital, Pakistan \\ Submission: May 18, 2018; Published: May 25, 2018 \\ *Corresponding author: Syeda Beenish Bareeqa, Medical Student, Jinnah Medical and Dental College, 21-22 Shaheed-e-millat road, Karachi, \\ Pakistan, Tel: (+92)342-2410338; Email: syedabeenishbareeqa@gmail.com
}

\begin{abstract}
Background: Preventive effect of leisure time physical activity on CHD, hypertension and diabetes has remarkable significance. Professional education is more time consuming. The available literature on the status of leisure time physical activity in students of professional institutions is scarce. This observation became the stimulus for this study.
\end{abstract}

Objective: We conducted this survey to evaluate the physical activity among young students of professional institutions. In addition, family history of co-morbid conditions like diabetes etc, stress level, sleep hours, smoking etc have also been observed.

Material and Methods: Our study was a prospective observational cross sectional study which was conducted at three leading professional institutes of Karachi. The data was collected through designed questionnaire based upon Global physical activity questionnaire of WHO. The inclusion criterion of our study was the inclusion of participants of 17-27 years of age. The exclusion criteria included all those students who were physically handicapped.

Results: In our study, total of 376 participants voluntarily took part in the survey. According to Global Physical Activity QuestionnaireWorld Health Organization (GPAQ-WHO), 121(32.2\%) individuals among the population were found to be physically inactive, 93(24.7\%) were moderately active, $92(24.5 \%)$ were moderately inactive and 70(18.6\%) were physically active. There was considerable association found between gender and physical activity with p-value of 0.026 , which is significant, with males being more physically active.

Conclusion: It was concluded that most of the students in professional institutions are physically inactive with females being more inactive as compared to males.

Keywords: Physical Activity; Young Adults; GPAQ-WHO; Basal Metabolic Index (BMI)

\section{Introduction}

According to world health organization (WHO), physical activity is defined as "Any bodily movement produced by skeletal muscles that require energy expenditure."An extensive review of population who belonged to a developed country suggested that $51 \%$ of the elderly population and only $27 \%$ of young adults meeting the aerobic components of guidelines for daily physical activity [1]. Whereas prevalence of physical activity in elderly population was significantly higher $(68.6 \%)$ in developing country than those in developed country. Negative association of prevalence of physical activity with high monthly income was also found in this cross-sectional study [2]. Regarding CHD a previous study indicates that physical activity, easily within the ability of almost all women, is associated with lower CHD rates. At least one hour of walking per week, regardless of pace, was associated with lower CHD rates among relatively sedentary women [3].

A cohort study conducted on young Finnish population concluded that male subjects tend to be more active than female subjects. Despite the fact, decline in physical activity 
in adolescence was steeper in boys as compared to girls. However, females in Finland tend to participate more regularly in physical activities after the age of fifteen as compared to male population [4]. here has been substantial debate about the volume expenditure required for physical fitness. Currently, most of studies suggest that $1000 \mathrm{kcal}$ per week is an optimal volume of energy expenditure. Regular physical activity results in primary and secondary prevention of several chronic illnesses (e.g., cardiovascular disease, diabetes, cancer, hypertension, obesity, depression and osteoporosis) and premature death [5]. Physical activity and physical fitness are closely related in that physical fitness is mainly, although not entirely is determined by physical activity patterns over recent weeks or months. Genetic contributions to fitness are important but probably account for less of the variation observed in fitness than is due to environmental factors, principally physical activity [6]. For most individuals, increases in physical activity produce increases in physical fitness, although the amount of adaptation in fitness to a standard exercise dose varies widely and is under genetic control [7]. At this time, it was speculated that inactivity might play a more important role than diet in the development of obesity. Several intervention studies have evaluated the impact of increased physical activity or decreased sedentary behavior as part of multi-factorial interventions on prevention of weight gain or remission of overweight in clinical and community-based settings. According to World Health Organization, inactivity is one of the ten major causes of mortality in the world. At least 30 min of moderate physical activity per day is adequate to achieve healthy life [8].

According to a study conducted in Minnesota, regular physical activity can help to prevent or manage a variety of chronic diseases and conditions including cardiovascular disease, osteoporosis, diabetes, hypertension, obesity, depression and anxiety. More lives are lost each year to physical inactivity and diet than to any other factor except tobacco use [9]. The prevalence of obesity among U.S. adolescents has increased dramatically in recent decades. Among young adults, physical activity is thought to be a key factor influencing insulin resistance and the future risk of developing type-II diabetes. In a study including white and African-American children, physical activity was independently associated with insulin sensitivity and secretion [10]. Objective of our study is to assess the physical activity in young adults who belong to different professional institutes.

\section{Material and Methods}

Our study was a prospective cross-sectional survey which was conducted at Jinnah medical and dental college, Institute of business administration and KNS institute of business studies. The duration of the study was 2 months dated from 1 st September 2016 to 1 st November 2016. The sample size was calculated and sampling technique used was non probability. In a total sample size of 377 students who participated in our study, 207 were enrolled in Jinnah medical and dental college, 119 were students of Institute of business administration and 51 participant studies at KNS institute of business studies.

The inclusion criterion of our study was the participants aged between 17 to 27 years, both male and female students who were currently enrolled as a student in the respective institutes. Participants younger than 17 years and older than 27 years of age were excluded from our study. We also excluded any handicapped and disabled students. The data was collected by trained medical students. The demographic data was collected through a designed questionnaire whereas physical activity was calculated with the help of preformed Global physical activity questionnaire. Other relevant questions were also included in formulated questionnaire.

Global physical activity questionnaire comprises of three domains with multiple response options. The collected information was entered by typing in that data on MS Excel spreadsheet. MS Excel spreadsheet was coded to give an automatic answer according to the given responses. The coding guidelines for MS Excel spreadsheet were mentioned on GPAQWHO website page. We followed the instructed guidelines to code the Excel sheet. As a result of appropriate coded spreadsheet, four different responses which were Active, Moderately active, Moderately inactive and Inactive were obtained.

Other variables of our designed questionnaire includes Age, Gender, Weight in kgs, Waist Circumference in inches, BMI, Institution, Family history of CVD, Diabetes, Cancer and Stroke, Smoking history, Sleep hours. Stress level of participants was assessed using tested questionnaire. Blood glucose level (random blood sugar) and Blood pressure were checked using digital Glucometer and manual BP apparatus respectively. Readings were taken and recorded by trained medical students.

The data was entered and analyzed on IBM statistics SPSS version 21 . The descriptive data was analyzed and associations among qualitative variables were also observed using chi-square test of association.

\section{Results}

In our study, total of 376 participants among which 156 (41.5\%) were males and 220 (58.5\%) were females. Data was collected from three different educational institutions. The quantitative description of participants is given in Table 1. According to results obtained from Global Physical Activity Questionnaire of World Health Organization (GPAQ-WHO), $121(32.2 \%)$ individuals among the population were found to be physically inactive, $93(24.7 \%)$ were moderately active, $92(24.5 \%)$ were moderately inactive and $70(18.6 \%)$ were physically active. Regarding smoking, 244(64.9\%) participants responded as non-smokers, 54(14.4\%) were ex-smokers, $58(15.4 \%)$ of all smoke less than 20 cigarettes/day and $20(5.3 \%)$ were current smokers who smoke more than 20 cigarettes/day. 
Table 1: Statistics of quantitative variables.

\begin{tabular}{|c|c|c|c|c|}
\hline & Mean/Median & SD/Intercortile & Maximum Value & Minimum Value \\
\hline Age & 22 & \pm 3 & 27 & 17 \\
\hline Weight in kg & 65 & \pm 21 & 193 & 137 \\
\hline Height in cm & 164 & \pm 15 & 125 & 34 \\
\hline Waist Circumference in cm & 85 & \pm 10 & 38.36 & 11.24 \\
\hline BMI & 23.59 & \pm 4.54 & & \\
\hline
\end{tabular}

0-4 hours sleep was recorded in 39(10.4\%) participants, 5-6 hours/day sleep in 139(37\%) participants, 7-8 hours/day sleep in $132(35.1 \%)$ participants and more than 8 hours/day sleep in $66(17.6 \%)$ participants. Blood glucose levels were low in $20(5.3 \%)$, normal in 351(93.3\%) and high in 5(1.3\%). Regarding blood pressure, it was low in $40(10.6 \%)$, normal in $150(84.5 \%)$ and high in $18(4.8 \%)$.

Regarding stress level, we measured anxiety levels on categorical basis. For anxiety, 35 (9.3\%) participants don't feel anxious, 145(38.6\%) individuals feel anxious weekly or more, while 196(52.1\%) individuals have monthly episodes of anxiety. For sadness and depression, individuals with negative answer were 33(8.8\%), 165(43.9\%) participants have these feeling weekly or more while, 178(47.3\%) of them have these monthly or more. Same had done for measurement of anger and frustration. 43(11.4\%) individuals doesn't feel angered or frustrated. $178(47.3 \%)$ have these feelings on weekly basis or more while, $155(41.2 \%)$ have these feelings on monthly basis or more.
Regarding CVD family history, 183(48.7\%) had no significant history of CVD, 99(26.3\%) individuals have CVD history of grandparents, 48 (12.8\%) individuals had paternal CVD history. Regarding familial diabetes, $139(37 \%)$ participants have no significant family history. $78(20.7 \%)$ participants had paternal diabetes history, grandparents of $70(18.6 \%)$ participants and mothers of $37(9.8 \%)$ participants have significant history of diabetes. Out of all participants, 291(77.4\%) had no family history of cancer, $42(11.2 \%)$ had cancer history in grandparents, $25(6.6 \%)$ had maternal history and $12(3.2 \%)$ individuals had paternal cancer history. Regarding stroke, 320(85.1\%) participants had no family history. Grandparents of $26(6.9 \%)$ participants, $14(3.7 \%)$ had paternal and siblings of $9(2.4 \%)$ participants have a significant history of stroke.

There was significant association found between gender and physical activity with males being more active than females $(\mathrm{p}=$ 0.026). The distribution of gender in relation to physical activity is given in Figure 1.

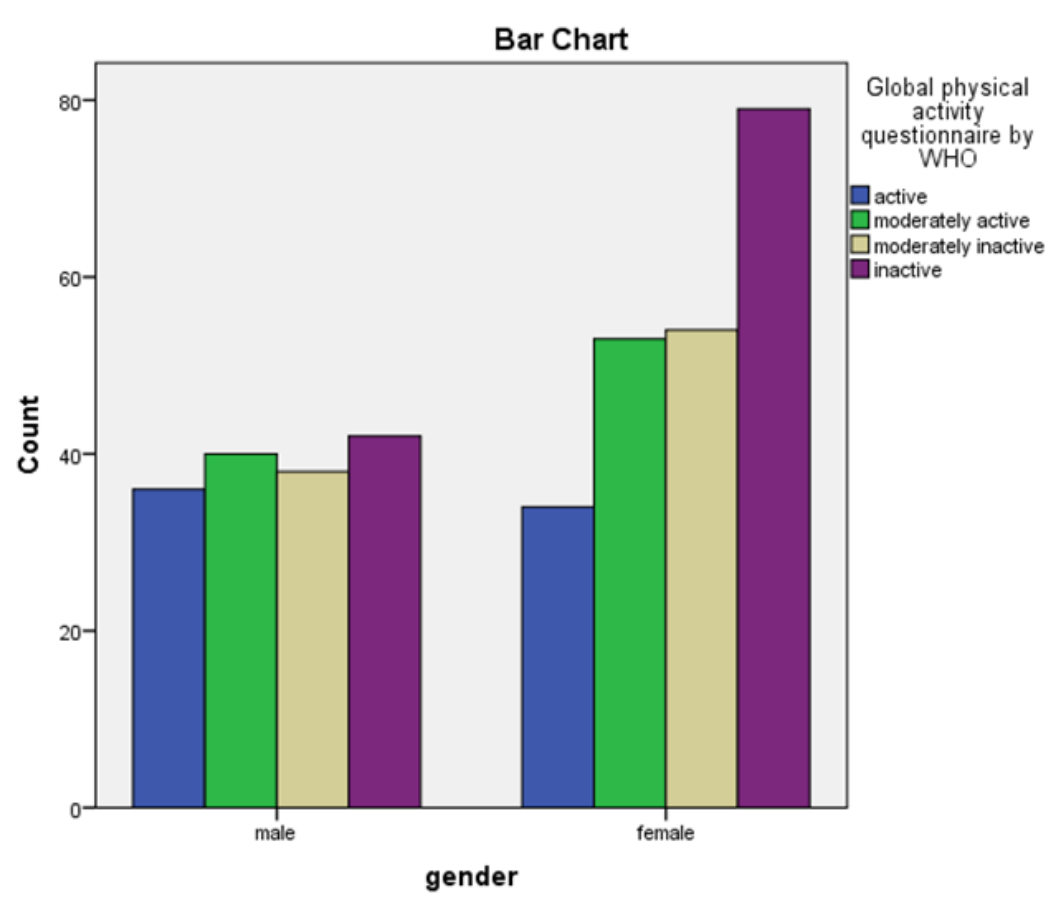

Figure 1: Distribution of gender in relation to physical activity. 


\section{Discussion}

The idea of developing the World Health Organization global physical activity questionnaire (GPAQ) was to have a tool that would produce valid and reliable estimates of physical activity, especially relevant to developing countries, like our country where the ways of energy expenditure differ from developed countries because people experience different lifestyles.

According to a study by Armstrong $\mathrm{T}$ and associates demonstrated that approximately 50 developing countries are now using GPAQ for the data collection for physical activity assessment. GPAQ is a suitable and reliable physical activity surveillance tool for developing countries [11]. This instrument was also used in our study which is proven to be an effective tool for the assessment of physical activity in developing countries like Pakistan. Higher levels of physical activity are associated with lesser cardiovascular disease (CVD) events. There is a inverse relation between physical activity and CVD risk in presence of known risk factors, particularly inflammatory/ haemostatic factors and blood pressure [12]. Our study has shown the familial risk for CVD in our participants and above study shown the improvement of CVD events among physically active people therefore physical activity should be promoted in those who have familial history of CVD. Sedentary students when become physically active, they reduce the risk of CVS events. Exercise benefit is independent of life-style elements such as smoking, obesity, weight gain, hypertension, and adverse parental disease history, in affecting coronary heart disease incidence. Hypertension is clinically the strongest predictor of coronary attack, but inadequate exercise is strongest on a community basis. A study by Paffenbarger RS shows that an exercise level is inversely related to the total, cardiovascular, and respiratory mortality but less related to unnatural deaths such as cancer. The current exercise revolution may impart positive effects on life-style, cardiovascular health, and life span [13]. Our study helped in the assessment of physical activity among young group of participants who are at risk of disorders discussed in the study mentioned above. Obesity and family history of diabetes are both important risk factors for developing type-II diabetes. It is noteworthy that even the modest loss in weight is effective in reducing the risk of type-II diabetes. Weight loss of only $4.5 \mathrm{~kg}$ ( $4.5 \%$ of the participant's initial body weight) reduced the risk of diabetes by $30 \%$ [14]. Increasing the total amount of physical activity in sedentary and overweight individuals may have beneficial effects on metabolic risk factors [15]. In our study, we assessed the population of comparatively young individuals to observe the risk of developing diabetes and obesity in near future.

An international study by Shinton $\mathrm{R}$ et al stated that remarkable protection from stroke is associated to increased levels of physical activity among individuals who have family history of stroke. According to this study increasing periods of vigorous physical activity between age 15-55 years increases protection from stroke [16]. As discussed in previously mentioned study, physical activity has protective effects on decreasing incidence of stroke so our study also emphasizes on physical activity in reducing risk for stroke in individuals with familial history.

Giovannucci E supports an inverse association between physical activity and risk for colon cancer, whereas height and obesity, particularly abdominal adiposity, are associated with an elevated risk [17]. Bernstein L and associates discussed the protective effect of exercise on breast cancer risk in the women whom they studied suggests that physical activity offers one modifiable lifestyle characteristic that may substantially reduce a woman's lifetime risk of breast cancer [18]. Physical activity also has remarkable effects on decreasing incidence of cancer as mentioned above and our study also explains the importance of physical activity in reducing incidence of cancer with any previous familial history.

In a study by Ferrucci L et al. [19], effects of smoking and physical activity on active and disabled life expectancy were estimated. The smokers who had good physical activity were found to have greater life expectancy than smoker with sedentary life style. Their findings provide strong and explicit evidence that refraining from smoking and doing regular physical activity predict a long and healthy life [19]. Our study also estimated the physical activity in relation to smoking habits among participants. In an international study relationship between physical activity and regular physical exercise has been characterized as a positive health behavior having physiological benefits. It may also yield psychological benefits. Their results indicate consistent association between enhanced psychological well-being, as measured using a variety of psychological inventories, and regular physical exercise [20]. As discussed in previous study, physical activity in beneficial for health. Naylor E et al demonstrated that low intensity activity in an elderly population can increase deep sleep and improve memory functioning. The high degree of interest in these activities paired with the simple nature of the tasks makes this a potentially practical intervention which can be adapted for both community dwelling and assisted-living elder [21] Our study assessed the effects of physical activity on sleep hours in young students.

In a clinical trial conducted by Arroll B and associates, all activities including circuit weight training, lowered blood pressure and daily activity produced greater blood pressure reduction than when performed three times per week. It was concluded that physical activity has an independent capacity to lower blood pressure. [22]. For those individuals, who have familial history of CVD in our study, physical activity has protective effects on decreasing incidence of hypertension or any CVD so our study also emphasizes on physical activity in reducing risk for hypertension or CVD in individuals with familial history. 
Few studies have examined cancer-related risk factors in relation to SES across the life course in low to middle income countries. This analysis focuses on adult women in India, China, Mexico, Russia and South Africa, and examines the association between individual, parental and life course SES with smoking, alcohol, BMI, nutrition and physical activity. Data on 22,283 women aged 18 years and older were obtained from the 2007 WHO Study on Global Aging and Adult Health (SAGE). Low SES women were almost four times more likely to exceed alcohol use guidelines. Cancer-related risk factors varied significantly by life course SES, suggesting that cancer prevention strategies will need to be tailored to specific sub-groups in order to be most effective [23].

During physical activity, whole-body oxygen consumption may increase by as much as 20 -fold and even greater increases may occur in the working muscles. To meet its energy needs under these circumstances, skeletal muscle uses, at a greatly increased rate, its own stores of glycogen and triglycerides, as well as free fatty acids (FFAs) derived from the breakdown of adipose tissue triglycerides and glucose released from the liver. The importance of promoting physical activity as a vital component of the prevention as well as management of type 2 diabetes must be viewed as a high priority. Therefore it is concluded that increased physical activity has a good impact of blood glucose regulation [24].

As mentioned previously, adequate physical activity is preventive for health related issues like diabetes and obesity. A study from New England journal of medicine assessed the effects of changes in diet and exercise, alone and together, on plasma lipoproteins. they hypothesized that HDL cholesterol levels would be raised by exercise but lowered by the NCEP Step 2 diet, that LDL cholesterol levels would be reduced by the diet, and that the ratio of LDL to HDL cholesterol would be most improved by combining the diet with regular exercise [25]. As the above study emphasize that physical activity help to lower the LDL levels and improve HDL levels in the body. Our study assessed the physical activity in relation to co-morbid. The limitation of our study includes its application to the students of only specific age group, it was only a three center study and handicapped or disabled population wasn't included in our study.

\section{Conclusion}

In light of substantial evidence, it was concluded that young adult males were being more physically active as compared to young adult females however the overall activity status in young adults is not sufficient to maintain the proactive lifestyle.

\section{Suggestions}

We recommend study on similar topic with larger sample size and inclusion of more educational centers. Studies like this shouldn't be confined to particular institutes as it proves to be helpful for the assessment of risk for co-morbid in relation to the daily physical activity among general young population. In future, study should be done on working class and elderly as most of the co-morbid show strong association with increasing age. It is also recommended to conduct a similar study among the population of low socioeconomic status in future.

\section{Refernces}

1. Katzmarzyk PT (2017) Epidemiology of physical activity and exercise training in the United States. Progress in cardiovascular diseases 60(1): 3-10.

2. Oyeyemi AL (2013) Prevalence of physical activity among adults in a metropolitan Nigerian city: a cross-sectional study. Journal of epidemiology 23(3): 169-177.

3. Lee IM (2001) Physical activity and coronary heart disease in women: Is no pain, no gain passã@)? Jama 285(11): 1447-1454.

4. Telama R, X Yang (2000) Decline of physical activity from youth to young adulthood in Finland. Medicine \& Science in Sports \& Exercise 32(9): 1617-1622.

5. Warburton DER, CW Nicol, SSD Bredin (2006) Health benefits of physical activity: the evidence. Canadian medical association journal 174(6): 801-809.

6. Bouchard C, L Perusse (1994) Heredity, activity level, fitness, and health in Physical activity, fitness, and health: International proceedings and consensus statement. Champaign, IL: Human Kinetics.

7. Blair SN, Y Cheng, JS Holder (2001) Is physical activity or physical fitness more important in defining health benefits? Medicine and science in sports and exercise 33(6 SUPP): S379-S399.

8. Soleymani B (2003) Department of Biostatistics and Epidemiology, School of Health, Isfahan University of Medical Scienc, Isfahan, Iran. Journal of Research in Medical Sciences 8(2).

9. Lobeck C SHC Community Health Needs Assessment.

10. Imperatore G (2006) Physical activity, cardiovascular fitness, and insulin sensitivity among US adolescents. Diabetes Care 29(7): 15671572.

11. Armstrong T, F Bull (2006) Development of the world health organization global physical activity questionnaire (GPAQ). Journal of Public Health 14(2): 66-70.

12. Mora S (2007) Physical activity and reduced risk of cardiovascular events potential mediating mechanisms. Circulation 116(19): 21102118.

13. Paffenbarger RS (1984) A natural history of athleticism and cardiovascular health. Jama 252(4): 491-495.

14. Wing RR (1998) Lifestyle intervention in overweight individuals with a family history of diabetes. Diabetes care 21(3): 350-359.

15. Ekelund U, SJ Griffin, NJ Wareham (2007) Physical activity and metabolic risk in individuals with a family history of type 2 diabetes. Diabetes care 30(2): 337-342.

16. Shinton R, G Sagar (1993) Lifelong exercise and stroke. BMJ 307(6898): 231-234.

17. Giovannucci E (1995) Physical activity, obesity, and risk for colon cancer and adenoma in men. Annals of internal medicine 122(5): 327334.

18. Bernstein L (1994) Physical exercise and reduced risk of breast cancer in young women. Journal of the National Cancer Institute 86(18): $1403-1408$ 
19. Ferrucci L (1999) Smoking, physical activity, and active life expectancy. American Journal of Epidemiology 149(7): 645-653.

20. Hassmen P, N Koivula, A Uutela (2000) Physical exercise and psychological well-being: a population study in Finland. Preventive medicine 30(1): 17-25.

21. Naylor E (2000) Daily social and physical activity increases slow-wave sleep and daytime neuropsychological performance in the elderly. Sleep 23(1): 87-95.

22. Arroll B, R Beaglehole (1992) Does physical activity lower blood pressure: a critical review of the clinical trials. Journal of clinical epidemiology 45(5): 439-447.

This work is licensed under Creative Commons Attribution 4.0 License DOI: 10.19080/JOJPH.2018.03.555620
23. Akinyemiju T (2017) Lifecourse socioeconomic status and cancerâ€ related risk factors: Analysis of the WHO study on global ageing and adult health (SAGE). International journal of cancer 140(4): 777-787.

24. (2004) American Diabetes A Physical activity/exercise and diabetes. Diabetes care 27: s58-s62.

25. Stefanick ML (1998) Effects of diet and exercise in men and postmenopausal women with low levels of HDL cholesterol and high levels of LDL cholesterol. New England Journal of Medicine 339(1): 12-20.

\section{Your next submission with Juniper Publishers} will reach you the below assets

- Quality Editorial service

- Swift Peer Review

- Reprints availability

- E-prints Service

- Manuscript Podcast for convenient understanding

- Global attainment for your research

- Manuscript accessibility in different formats ( Pdf, E-pub, Full Text, Audio)

- Unceasing customer service

Track the below URL for one-step submission https://juniperpublishers.com/online-submission.php 